\title{
Ethical Justification of Conducting Research Trials in Lower and Middle Income Countries Including Pakistan: The Responsibilities of Research Enterprises
}

\author{
Zoheb Rafique \\ Lecturer Department of Biochemistry, \\ Liaquat University of Medical \& Health Sciences (LUMHS), \\ Jamshoro, Pakistan
}

Email: dr zohaib@hotmail.com

\begin{abstract}
Asia is the most diverse continent in the world in terms of culture, religion, population size, finance, education, health care, academic research, general population skills, and governmental drug regulations. Each Asian country has its own unique qualities when it comes to attracting industry sponsored clinical trials. Factors that influence selecting location of a study site for a sponsored trial are mainly population size, infrastructure, education levels, and quality of health care, cost and drug regulatory platform. Conducting research in traditional countries like Pakistan adds another dimension to the problems in assuring that research conducted in an ethical manner. The indigenous layer of the cultural values makes it even more difficult task, but this is the task that we are morally and ethically bound to shoulder.
\end{abstract}

Key words: Responsibilities, Research Enterprises, Research Trials Lower and Middle Income Countries, Pakistan

Introduction: Some Asian countries such as Japan, Hong Kong and Singapore have among the longest life-expectancy, lowest infant mortality and highest per capita income worldwide, while others are in the lower end of such rankings. Several, notably China and India, are amid rapid economic development, as the Asian economy is more-or-less becoming the global axis, with the economies of US and Europe slowing. Asia has a population of 3.8 billion, at least ten times more than North America or Europe. As the world's most populous continent, Asia has by no means reached full capacity in contributing with subjects in testing new medical products in collaboration with the international pharmaceutical industry. This trend will certainly direct more sponsored clinical trials to Asia, but not necessarily benefit all Asian countries. Engaged in 18.1 $\%$ of all protocols globally Asia is involved in more sponsored trials than any other region. India, Korea and Taiwan standout as the most active locations for multi-national trials in Asia. When ranking is for cities Seoul is the most active Asia city, followed by Taipei, Hong Kong, Singapore and New Delhi. The globalization process of sponsored clinical trials has provided an opportunity for Asia to attract international companies to the region and also seemingly encourages development of local life-science industries ${ }^{1}$. We are beginning to see some 
exponential growth in the clinical drug trials conducted in Pakistan in which multinational drug manufacturing companies are major players. In majority of collaborative trials the sponsors are from countries of developed world while co- investigators and research participants are drawn from Asian and African countries ${ }^{2}$.

In this paper, I will discuss the responsibilities of researcher/funder when the research trial is conducted in low and middle income countries like Pakistan and I will also talk on ethical justifications of doing research trials in Pakistan and other poor and developing countries.

Discussion: Resource poor countries require a lot of attention from the medical research establishment in order to sustain the quest for treatments and remedies for diseases and other health-threatening conditions. However, the collaboration between rich countries and wellendowed agencies, on one-hand, and economically constrained research communities, on the other, requires a careful assessment of responsibilities and options for researchers and research subjects alike. Major players in international research also include big pharmaceutical companies who seek people living in developing countries as subjects. Safety and standard of care for human volunteers are also major issues. "I had not been exploring Big Pharma of third world 'volunteers' as cheap guinea pigs, observes writer John le Carre (2001). "Their role though they don't even know this is to test drugs, which are not yet approved for the testing in US, and which they themselves never be able and possible to afford even if those tests turn out to be reasonably safe" (le Carre, 2001). In US, it costs on an average \$10,000 per participant to conduct one clinical trial, in Russia it costs $\$ 3,000$, and in poorest and remote parts of the world, it costs much less. This is among one of strong reasons why the clinical trials are now Third world growth industry. In its May 2000 edition Center Watch, a newsletter for burgeoning clinical trials business, published an article under title Latin American Fever, in which it said that the continent "may offer a unique chance to reach larger numbers of the study subjects". Eli Lilly tested some 590 patients, in 1994 across Africa, Middle East and the Central and Eastern Europe. In 2001 the company expected to run the tests in those regions on 7,309 patients. In rush to market, poorly constructed, and weakly monitored drug trials are releasing the untried and untested drugs. The Contemporary practice of biomedical research on a global scale has given rise to evolving forms of exploitation. Standards of justice and equality tend to be put in question in the face of research practices that often put heavy burdens on poor people and poor communities in poor countries. There is a need to remain vigilant in the prior review of these activities and the monitoring of their implementation in order to ensure that biomedical research is conducted in accordance with universally acceptable standards. One of the most important requirements for the conduct of research in developing countries is emphasized in the WHO-Council for International Organizations of Medical Sciences' Guidelines for Biomedical Research Involving Human Subjects: to guarantee that those communities where these new drugs have been tested will be given affordable access to the newly developed and approved drugs. Otherwise, one might 
rightly argue that people in developing countries have yet again been exploited by Western researchers without benefiting from the positive results their risk-taking has yielded (Del Rio, Kamarulzaman, and Schuklenk, n.d.). Ruth Macklin observes that it is not just individuals who can lose out when big drug companies carry out their tests. When industrialized countries do research in a developing country, the developing country can't afford the products of that research. The researcher's pullout and the successful products then become available in the Western industrialized countries and the population in the countries where the research was done get nothing. So that's truly a question of justice, and we're beginning to see a movement to rectify that injustice (2000). The wide disparities in resources that are available for biomedical research in developed and developing countries give rise to ethically relevant issues of research prioritization and collaboration. The international research community has to accelerate the shift to an environment where researchers from developing countries are recognized as full and equal partners in biomedical studies; where the technologies of developed and developing countries are integrated and made widely available; and where the benefits of biomedical research for participant communities can be ensured ${ }^{3}$. In resource-poor countries like Pakistan and majority of developing countries, the two primary means of protecting participants-IRB review and Informed Consent may be inadequate. IRBs in low and middle income countries may lack training, experience, and resources. IRBs in the United States are unlikely to be familiar with conditions in the host country. Informed Consent may be problematic in a country where people are poorly educated and lack health literacy, and where physicians in clinical practice usually do not tell patients their diagnosis, admit uncertainty, or obtain consent. Participants may not accept Western models of disease. Furthermore, participants might hear rumors and other misinformation about a research study. In several highly publicized cases, researchers from developed countries have been harshly criticized for allegedly conducting inappropriately risk studies in resource poor counties without adequate consent. The other problem is health priority and it would be an imprudent use of limited health care resources in a developing country to conduct human-participants research that does not address a health or public health priority in the host country. Because of scarce resources and logistical constraints, medical interventions that are standard in developed countries may not be available or feasible in resource poor countries where the trial is conducted. This creates an ethical tension between providing a benefit to research participants and obtaining generalizable scientific knowledge. According to the ethical obligation to minimize harm to participants, researchers should provide interventions that are known to be effective and feasible to prevent or treat the condition addressed in the clinical trial. Because participants in a research study help researchers, sponsors, and society at large, they should receive some benefit in return as a matter of reciprocity. Advocates contend that researchers and sponsors must avoid taking unfair advantage of participants and their communities by providing those who bear the risks of research appropriate benefits, in addition to the long-term benefit of generalizable knowledge. Researchers and Sponsors need to consider 
whether the study intervention will be available in the host country if it is shown to be effective and safe. Some ethics expert point out that providing reasonable access to study interventions after a trial may be an inadequate reciprocation for participation in research. First, it is too limited and weak an obligation. If the study is something other than a pivotal clinical trial (for example, an epidemiological study), no additional benefits will be required. Even if the study is a clinical trial, it might be a negative study. Second, other benefits might be more useful to participants or their communities than the trial drug. For example, they might benefit more from better primary care or better education for host country health care workers. Third, the appropriate target group for benefits may be all persons in the community where the study is carried out, not just trial participants. Providing benefits only to trial participants will widen health disparities in the resource-poor host country and therefore raise concerns about causing injustice. Thus, providing benefits to the host country should be done in a way that ameliorates rather than worsens health disparities. For these reasons, some writers argue that researchers and sponsors from the developed world should provide fair benefits to the research participants and their communities in reciprocity for what they contribute to the research. Researchers could provide benefits to research participants in a number of ways, such as by providing health education or some basic health services; training local health care workers, researchers, and IRBs; donating equipment at the end of the study; and giving local investigators a key in analyzing data and writing papers. Such contributions ensure that the community where the research is carried out will receive benefits in reciprocity for participating in the research. By building infrastructure, researchers can help provide sustainable improvements that will help to narrow health disparities between rich and poor nations ${ }^{4}$. Pakistan is also among the poor and one of developing country and the health care conditions here are same as any South Asian or African country. We will apply the same ethical and moral rules when we talk about research here in Pakistan. Added to this is other reality in Pakistan i.e. weak, absent and only-on-paper accountability processes both at governmental and institutional levels. The lack of accountability processes and also the powerful power difference serve as a lethal combination for the human research participants and patients alike. Through workshops held in our country Pakistan, healthcare professionals and medical scientists are now becoming familiar with Helsinki Declaration and the Council for International Organizations of Medical Sciences (CIOMS) and some other research ethics guidelines. But the knowledge of such guidelines is one thing and application of this within the specificity and the context of existing professional and cultural practices quite another. Ethical requirements for the clinical research don't end when subjects either sign consent form or when they are enrolled in the research or they refuse enrollment. The individuals should continue to be treated and healed with respect and empathy right from the time they are approached and even if they refuse enrollment throughout the participation, and even after the participation ends ${ }^{5}$. The basic disclosure requirement for satisfying the informed consent provision in U.S. research regulations focus on information needed by a potential 
participant to decide whether or not to participate in a study. Of the eight basic disclosure requirements, one focuses on potential benefits: a description of any benefits to the subject or to others which may reasonably be expected from the research. Traditionally disclosure is required to make sure that potential participants/subjects understand whether there is possibility that the procedure/intervention itself would benefit them when they are enrolled in research study. There is, however no any specific mention of any of post-trial benefits. Those who may participate in the studies should be informed about the potential benefits if any. Because this information is very relevant to the participants' decisions to involve in research, ethical review committees should require the investigators to make those disclosures ${ }^{6}$.

Conclusion: Biomedical research has provided huge benefits for the human, and it also offers good hope for the future. The problem arises when it is being high jacked by private and commercial enterprises those are willing to by pass the meticulous scientific methodology and the ethical standards. In the end I will conclude by saying that research participants should know each and every thing regarding their participation in the research trial or rejecting it, and it is their right to know all risks and benefits while participating in the research trials and this should be applicable to all countries around the world including all developing poor, middle and low income countries including Pakistan.

\section{References:}

1. Johan PE Karlberg. Development of Sponsored Clinical Trials in Asia. Clinical Trial Magnifier. 2008; Vol. 1:5: 77-100.

2. Farhat Moazam. Research and developing countries: hopes and hypes. East Mediterranean Health Journal. 2006; Vol. 12 (Supplement no. 1): 30-36.

3. 3. Leonardo D. de Castro Et Al. Bioethics in the Asia-Pacific Region: Issues and Concerns. 2003; 1-108.

4. LO B. Clinical Research in Resource-Poor Countries. Ethical issues in Clinical Research: A practical Guide. 2008; Ch.22:194-210.

5. Ezekiel J. Emanuel Et A1. What Makes Clinical Research Ethical? JAMA. 2000; 283(20): 2701-2711.

6. Ch 3; Voluntary Informed Consent. National Bioethics Advisory Commission. 35-53.

Conflict of interest: There is no conflict of interest 